\title{
ERRATUM
}

\section{Angiotensin II enhances the increase in monocyte chemoattractant protein-1 production induced by tumor necrosis factor- $\alpha$ from 3T3-L1 preadipocytes}

Sachie Asamizu, Masaharu Urakaze, Chikaaki Kobashi, Manabu Ishiki, Amal Khalifa Norel Din, Shiho Fujisaka, Yukiko Kanatani, Agussalim Bukahari, Satoko Senda, Hikari Suzuki, Yuh Yamazaki, Minoru Iwata, Isao Usui, Katsuya Yamazaki, Hirofumi Ogawa, Masashi Kobayashi and Kazuyuki Tobe

The authors apologise for an error in the name and affiliation of one of the co-authors in this paper that was published in 2009202 199-205.
Hirofumi Ogawa was wrongly spelt as Hiroshi Ogawa and his correct affiliation is Department of Molecular Neuroscience, Faculty of Medicine, Toyama University. 